Research Paper

\title{
The Therapeutic Effect of Human Adult Stem Cells Derived from Adipose Tissue in Endotoxemic Rat Model
}

\author{
Soyoung Shin ${ }^{1}$, Yonggoo Kim ${ }^{1}$, Sikyoung Jeong ${ }^{2}$, Sungyoup Hong ${ }^{2}$, Insoo Kim², Woonjeong Lee ${ }^{2}$, \\ Seungphil Choi ${ }^{2}$ \\ 1. Department of Laboratory Medicine, The Catholic University of Korea, College of Medicine, Seoul, Republic of Korea \\ 2. Department of Emergency Medicine, The Catholic University of Korea, College of Medicine, Seoul, Republic of Korea
}

$\square$ Corresponding author: Sikyoung Jeong, M.D. Ph D., Department of Emergency Medicine , College of Medicine, The catholic University of Korea, Daejeon St. Mary's Hospital, Daejeon 301-723, Republic of Korea. Telephone: +82-42-220-9115; Fax: +82-42-256-6810; E-mail: sikyoung@catholic.ac.kr

() Ivyspring International Publisher. This is an open-access article distributed under the terms of the Creative Commons License (http://creativecommons.org/ licenses/by-nc-nd/3.0/). Reproduction is permitted for personal, noncommercial use, provided that the article is in whole, unmodified, and properly cited.

Received: 2012.10.15; Accepted: 2012.12.02; Published: 2012.12.06

\begin{abstract}
Excessive systemic inflammation following sepsis, trauma or burn could lead to multi-organ damage and death. Bone marrow stromal cells (BMSCs), commonly referred to as mesenchymal stem cells (MSCs), has been studied in several immune-associated diseases in human and animal by modulating the inflammatory response. Adipose tissue derived mesenchymal stem cells (ATSCs), which can be obtained more easily, compared with BMSCs, has emerged as an attractive alternative MSCs source for cell therapy. We investigated the therapeutic effects of human ATSCs (hATSCs) in endotoxemic rat model and their capacity to modulate the inflammatory response. Endotoxemia was induced with Lipopolysaccaride intravenously injection (LPS, $10 \mathrm{mg} / \mathrm{kg}$ ). Animals were divided into the following three groups: (I) saline + saline $(n=5),(2)$ LPS + saline $(n=5)$ and (3) LPS + hATSCs $\left(2 \times 10^{6}\right)(n=5)$. The administration of LPS caused a consistent systemic inflammatory responses, increased concentrations of the pro-inflammatory cytokines that have an important role in sepsis. Treatment of endotoxemia with hATSCs decreased the level of inflammatory cytokines both in serum and in the lung, reduced inflammatory changes in the lung, prevented apoptosis in the kidney and improved multi-organ injury. In conclusion, our data demonstrates that hATSCs regulate the immue/inflammatory responses and improve multi-organ injury and they could be attractive candidates for cell therapy to treat endotoxemia.
\end{abstract}

Key words: Endotoxemia, Adipose tissue derived mesenchymal stem cells(ATSCs)

\section{Introduction}

The sepsis involves complex inflammatory responses between a microbial pathogen and the host immune system. The host defense mechanisms fail to block pathogen invasion, which leads to microbial proliferation, and the release of microbial products activates the host inflammatory responses[1]. In early stage sepsis, endogenous pro-inflammatory cytokines and coagulation pathways are hyperactive and out-of-balance, causing multi-organ failure, collapse of the circulatory system, and death[2]. This imbalance is rapidly compensated by an anti-inflammatory response that deactivates the major immune responses. The extent and duration of these multiple immune dysfunctions are related to morbidity and mortality, and the patients who survive sepsis are those who recover normal immune functions[3]. 
Several clinical trials to control sepsis have modulated these complex inflammatory responses, by neutralizing cytokines with monoclonal antibodies or blocking the receptor of an inflammatory cytokine[4,5]. However, these attempts, which focusing on a single pathway, have had limited efficacy. Mesenchymal stem cells (MSCs) function at several levels of the inflammatory response, especially in the early stage of sepsis, to regulate a wide panel of inflammatory cytokines and inhibit leukocyte infiltration into several target organs[6,7]. Therefore, cell therapy has recently been explored as a new modality to regulate immune responses $[8,9]$.

Apart from BM-MSCs, MSCs have been also identified in several other accessible tissues, including umbilical cord blood, peripheral blood, and adipose tissue [10-12]. Several studies of bone marrow-derived stem cells (BMSCs) have been reported to treat inflammatory diseases[13-17]. However, bone marrow harvest is a painful procedure and low numbers of MSCs are present in the harvested marrow, which can be impose a load on some patients with severe complication[11]. Compared with other tissues, large amounts of adipose tissue can be obtained from lipoaspirates and also contain high numbers of MSCs that can be easily expanded in vitro to generate clinically effective dosages[19]. Because of this, hATSC has become an attractive alternative source of MSCs for cell therapy. Moreover, several studies have demonstrated that adipose tissue stem cells (ATSCs) share some immunomodulatory properties with BMSCs[11,18,19]. We wondered whether human ATSCs (hATSCs) could bring disrupted inflammatory responses back into balance, thus improving the underlying pathophysiology that progresses to severe sepsis, septic shock, and death. Therefore we investigated the therapeutic effects of hATSCs in endotoxemia.

\section{Materials and Methods}

\section{Preparation of hATSCs}

Subcutaneous adipose tissues were acquired from elective surgeries with patient consent as approved by the Institutional Review Board of the Catholic University of Korea, College of Medicine. Ten grams of adipose tissue was stored in serum-free DMEM/F12 medium (Gibco-BRL). The adipose tissue was then washed thoroughly with PBS (PBS, Sigma-Aldrich, St. Louis, MO, USA) and mechanically chopped before processing. To isolate the stromal vascular fraction (SVF), the tissue was enzymatically digested with serum-free DMEM/F12 medium containing $0.1 \%$ collagenase A (Sigma Aldrich) for 30 to
60 minutes at $37^{\circ} \mathrm{C}$ with continuous shaking. The digested tissue was then washed with DMEM/F12 medium containing $10 \%$ fetal bovine serum (FBS), and red blood cells were lysed with $3 \mathrm{ml}$ of red blood cell lysing buffer (Sigma) for 5 minutes. Next, $47 \mathrm{ml}$ of DMEM/F12 medium containing 10\% FBS was added, and the cells were applied to a $40 \mu \mathrm{m}$ cell strainer BD, Falcon, Franklin Lakes, NJ, USA). The cells were centrifuged for 10 minutes at $200 \mathrm{~g}$ to remove blood and unnecessary tissue. The cell pellet was washed twice in DMEM/F12 medium containing 10\% FBS. The SVF cell pellet was seeded onto a conventional tissue culture flask in DMEM/F12 supplemented with $10 \%$ FBS, $1 \%$ penicillin, $100 \mu \mathrm{g} / \mathrm{mL}$ streptomycin, $2 \mathrm{mM}$ L-glutamine (Gibco-BRL, Sigma Aldrich ), in a $37^{\circ} \mathrm{C}$ incubator with 5\% CO2. After $24 \mathrm{~h}$ of culture, non-adherent cells were removed. The media was changed every other day. When the monolayer of adherent cells reached $90 \%$ confluence, the cells were sub-cultured using $0.05 \%$ trypsin-EDTA (Gibco-BRL). For this study, cells were used after 5 passages, and cell viability was assessed using the trypan blue exclusion assay with $0.2 \%$ trypan blue.

\section{Phenotypic Characterization of hATSCs}

hATSCs were suspended and washed twice in PBS by centrifuging at $200 \times \mathrm{g}$ for $10 \mathrm{~min}$ at $4^{\circ} \mathrm{C}$. To inhibit unnecessary binding of primary antibodies, the cells were treated with PBS ( $\mathrm{pH} 7.5)$ supplemented with $1 \%$ bovine serum albumin (BSA, Bovogen, Biologicals Pty, Australia) for $30 \mathrm{~min}$ at $4^{\circ} \mathrm{C}$. Cells were then incubated with antibodies (CD29-FITC, CD34-FITC, CD105-FITC; Invitrogen, CD90-FITC; BD) for $30 \mathrm{~min}$ at room temperature. Fluorescence activated cell sorter (FACS) analysis was performed using a FACSCantoII flow cytometer (BD Biosciences, Germany). Gated events $\left(2 \times 10^{4}\right)$, except doublets and aggregates, were acquired for each sample and analyses were carried out with FlowJo ${ }^{\circledR}$ and FACSdiva software.

\section{Induction of Endotoxemia with LPS and hATSCs Administration}

Endotoxemia was induced in male Sprague-Dawley rats weighing 250-320 g. All interventions and animal care were provided in accordance with the Laboratory Animals Welfare Act, the Guide for the Care and Use of Laboratory Animals, and the Guidelines and Policies for Rodent Survival Surgery provided by the Institutional Animal Care and Use Committee at the Catholic University of Korea School of Medicine. All rats had free access to food and water, both before and after the procedure.

Rats were intraperitoneally anesthetized with 30 
mg/kg Zolazepam (Zoletil 50, 25mg/ml, Virbac Animal Health Inc., France) and $10 \mathrm{mg} / \mathrm{kg}$ xylazine hydrochloride (Rompun, $23.32 \mathrm{mg} / \mathrm{ml}$; Bayer Inc., Toronto, Canada). To induce endotoxemia, lipopolysaccharide (LPS extracted from Escherichia coli 0127: B8, Sigma-Aldrich, St Louis MO; $10 \mathrm{mg} / \mathrm{kg}$ ) was intravenously injected via the tail vein. A sham intervention was performed using the same amount of saline in control rats. Thirty minutes after administering LPS, either hATSCs $\left(2 \times 10^{6}, 100 \mu \mathrm{l}\right)$ or saline $(100$ $\mu 1)$ was slowly injected into the tail vein over $20 \mathrm{~min}$. Rats were randomly assigned to one of three experimental groups: 1) saline solution plus saline treatment $(n=5), 2)$ LPS plus saline treatment $(n=5)$, and 3) LPS plus hATSCs treatment $(n=5)$. During all procedures, rat body temperatures were maintained at $36.5 \pm 0.5^{\circ} \mathrm{C}$ with a heating pad (FHC, Bowdoinham, ME, USA).

\section{Serum and Tissue Collection}

Six hours after LPS challenge, the rats were sacrificed to collect serum and tissue. Blood was collected through cardiac puncture and centrifuged at 2,000 $\mathrm{xg}$ for $20 \mathrm{~min}$ to obtain the serum. Serum was stored at $-70^{\circ} \mathrm{C}$ to determine cytokine levels and analyze biomarkers. The right lung was removed en bloc and fixed in $10 \%$ buffered formalin (Sigma-Aldrich) for histopathology. The left lung was frozen at $-70^{\circ} \mathrm{C}$ to determine cytokine levels. Both kidneys were fixed in $10 \%$ formalin for $24 \mathrm{~h}$ before TUNEL staining.

\section{Cytokine Measurement in the Serum and Lung}

Levels of the cytokines, TNF- $a$, IL- 6 , and IL-10, in the serum and lung homogenates were measured by enzyme-linked immunosorbent assay (ELISA) using rat-specific kits (Thermo Scientific, Rockford, IL, USA). The lung was homogenized in ProPrepTM Protein Extraction Solution (iNtRON Biotechnology, Seongnam, Korea) and centrifuged for $10 \mathrm{~min}$ at $12,000 \mathrm{rpm}$. Equal amounts of protein were used for ELISA. ELISA was performed according to the manufacturer's instructions. All samples and standards were measured in duplicate.

\section{Measurement of Biochemical Markers for Organ Dysfunction}

Organ dysfunction was determined by measuring biochemical indicators of organ function in serum samples taken 6 hours after LPS challenge. Serum levels of blood urea nitrogen and creatinine (indicators of renal dysfunction), aspartate aminotransferase (AST) and alanine aminotransferase (ALT) (indicators of hepatic dysfunction), and lactate (an indicator of tissue hypoperfusion) were measured by IDEXX VetTest (IDEXX Laboratories, Maine, US).

\section{Histopathology Examination}

Lungs from each group were taken for histopathology. The trachea was incised, and a 14-G tube was placed inside. The left lung was ligated with 3-0 silk, and the right lung was fixed by inflation with $10 \%$ buffered formalin solution and removed en bloc. Following overnight fixation, the right lung was embedded in paraffin. The specimen was sliced into $4-\mu \mathrm{M}$-thick sections and stained with hematoxylin and eosin. Images were taken under a Nikon Eclipse E800 microscope with a 40× objective. Two independent experts blinded to the treatment accessed the Acute Lung Injury Score[20], which comprises 4 components (alveolar capillary congestion, hemorrhage, inflammatory cells infiltrating the airspace or interstitium, and thickness of the alveolar wall) each scored on a 5 -point scale $(0=$ minimal damage, $1+=$ mild damage, $2+=$ moderate damage, $3+=$ severe damage, $4+=$ maximal damage). The total injury score was the sum of all component scores.

The kidney was fixed with 10\% formalin for 24 hours and embedded in paraffin. Sections were examined for terminal deoxynucleotidyl transferase dUTP nick end labeling (TUNEL) staining with an in situ cell death detection kit (Millipore). TUNEL-positive cells were counted from 4 randomly chosen fields per slide by an investigator, who was blinded to the identity of the slides.

\section{Statistical analysis}

Statistical analysis was performed with the Stata/SE program 11.0 (StataCorp LP, College Station, TX). Comparisons among the three groups were made by Kruskal-Wallis test, with $\mathrm{p}<0.05$ used to reject the null hypothesis. The Bonferroni correction was used to assess differences between two groups, and a value of $p<0.017$ was considered statistically significant. The bar on the each graph was expressed as mean \pm standard deviation.

\section{Results}

\section{hATSCs Culture and Phenotypic Characteri- zation}

After the third passage, hATSCs appeared as a monolayer of flat cells. hATSCs appeared to be regular-sized, spindle-shaped, and had a fibroblastic morphology in culture, which was consistent with mesenchymal stromal cells[21](Fig 1A). hATSCs were 95\% viable, as determined by the trypan blue exclusion assay.

The surface markers expressed by hATSCs were determined by FACS to determine the purity of the population. The results demonstrated that the major- 
ity of hATSCs expressed MSC surface antigens, such as CD29 (90.8\%), CD90 (98.5\%) and CD105 (39.7\%). and were negative for the hematopoietic stem cell marker, CD34 (<1\%)(Fig 1B). hATSCs were classified as mesenchymal stem cells.

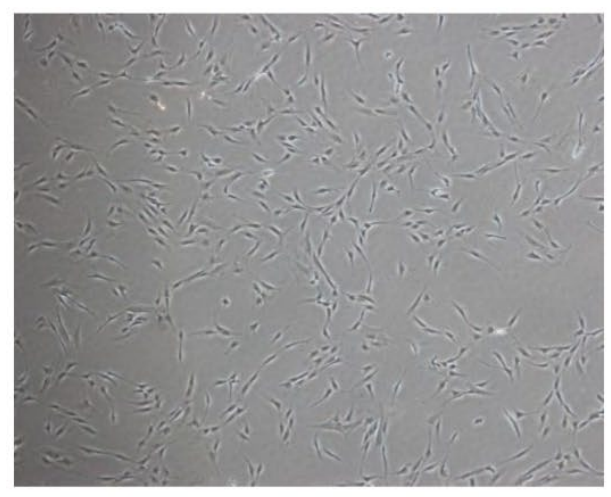

A. Morphology of hATSCs
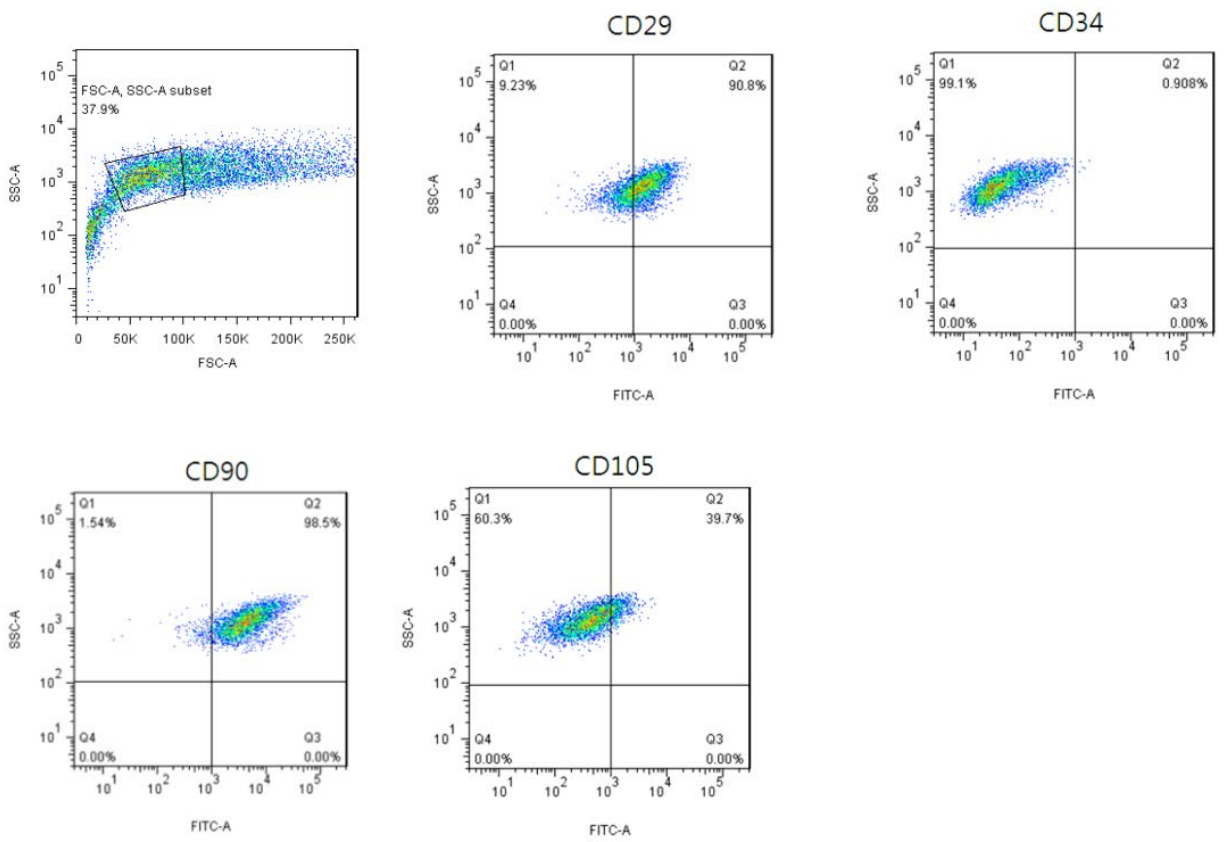

\section{B. Phenotypic Characterization of hATSCs.}

Figure I. A. hATSCs appeared to be of a regular size, spindle shape, and fibroblastic morphology in culture, which is consistent with mesenchymal stromal cells. (×40) B. Surface marker expression was determined by FACS. The majority of hATSCs expressed the surface antigens of mesenchymal stem cells (CD29, CD90 and CDI05) and were negative for the hematopoietic stem cell marker (CD34). 


\section{hATSCs Reduce Systemic Inflammation in Endotoxemic Rats}

Rats were randomly assigned to receive injections of saline or hATSCs into a tail vein 30 mins after endotoxemia was induced with LPS. We measured cytokine concentrations in the serum and lung tissue from all groups $6 \mathrm{~h}$ after adminstering LPS. Figure 2 summarizes the serum and pulmonary cytokine levels. Compared to control rats, LPS consistently caused systemic inflammatory responses, such as increased concentrations of the pro-inflammatory mediators,
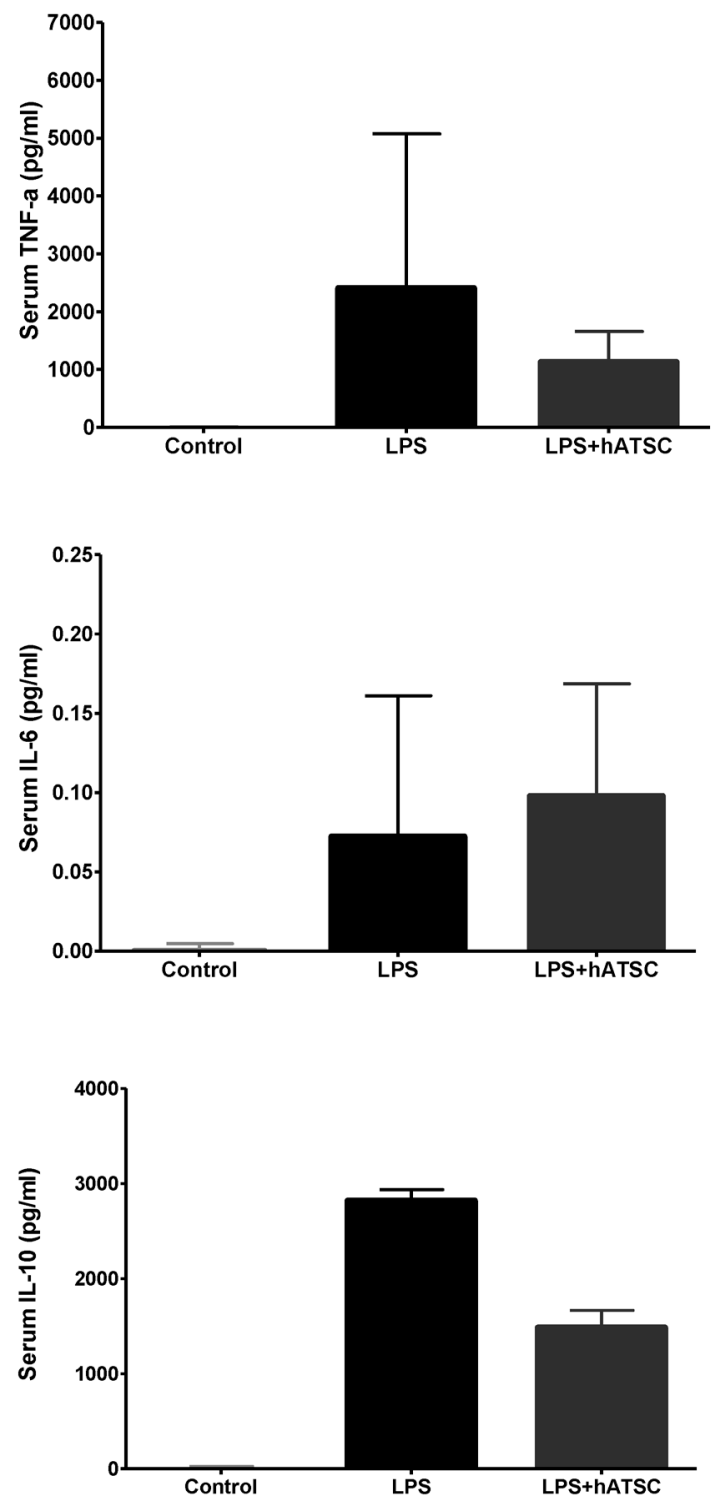

TNF-a and interleukin-6 (IL-6), which have central roles in sepsis. hATSCs modulated the increase of these pro-inflammatory mediators in serum and in the lung tissue, especially decreasing the pulmonary IL-6 level $\left({ }^{*} p<0.017\right)$, compared to controls. Both serum and pulmonary TNF-a levels tended to decrease in the hATSC group, compared to the LPS group. Serum levels of the anti-inflammatory cytokine IL-10 in LPS group tended to be higher than hATSC rats, although the difference was not significant. Pulmonary IL-10 was not significantly different among all 3 groups.
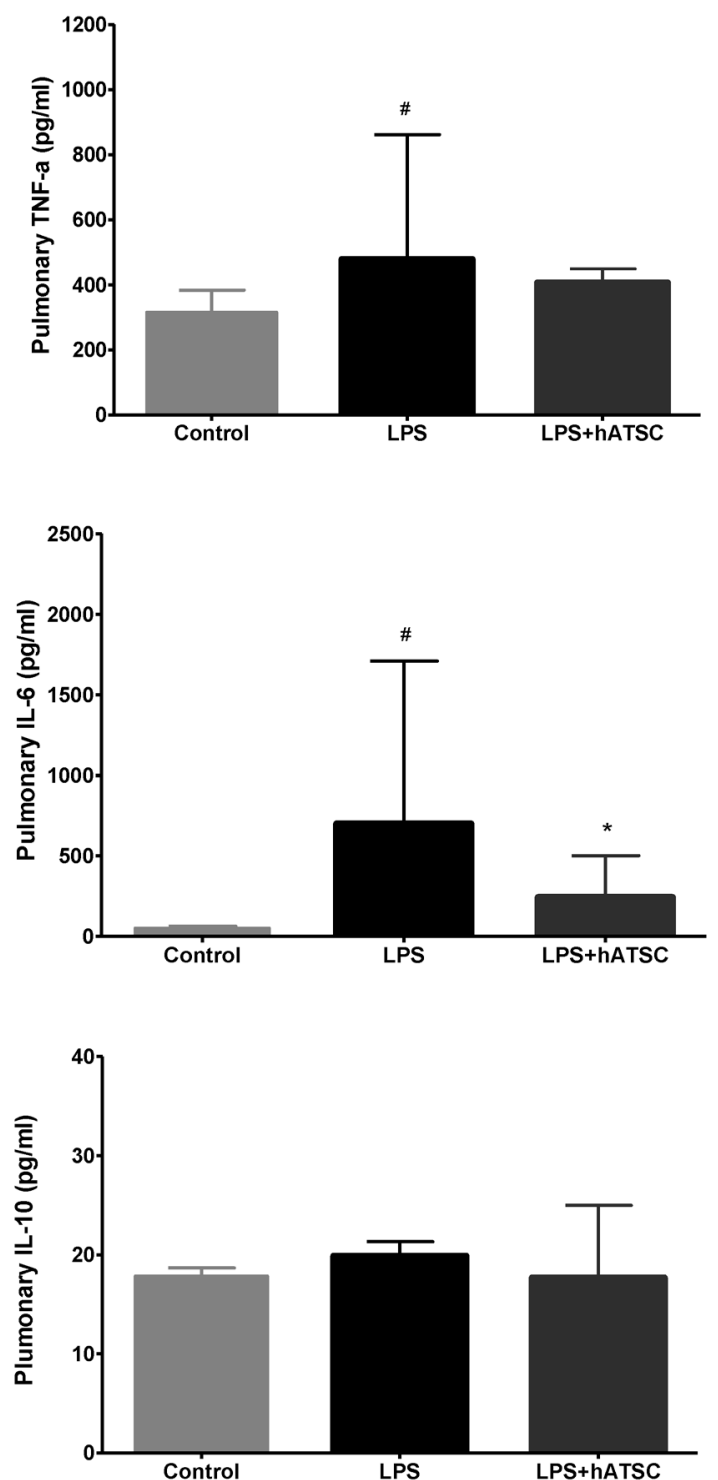

Figure 2. Cytokine levels in the serum and lung. Compared with control rats, LPS increased concentrations of the proinflammatory mediators, TNF-a, and interleukin (IL)-6. hATSC treatment modulated the increase in proinflammatory mediators, especially decreased pulmonary IL-6 compared with the LPS group $\left({ }^{*}<<0.017\right)$. Systemic levels of IL-10, an anti-inflammatory cytokine, were higher in the LPS group than the hATSC-treated group, though the difference was not statistically significant. Pulmonary IL- 10 did not differ among the three groups. $\# \mathrm{P}<0.017$, sham/saline versus LPS/saline group. ${ }^{*} \mathrm{P}<0.017$, LPS/saline versus LPS/hATSC group. $\mathrm{n}=5$ per group. The bar on each graph expresses mean \pm standard deviation. 


\section{hATSCs Treatment Improves Multi-Organ Dysfunction induced by LPS}

Because the lethality of sepsis is associated with organ failure, we examined the dysfunction and pathology of major organs often injured during sepsis. Organ dysfunction was determined by measuring biochemical indicators in serum samples taken 6 hours after administering LPS. LPS increased the levels of each biomarker, which demonstrated that LPS induced multi-organ dysfunction (Fig 3). Concentra-
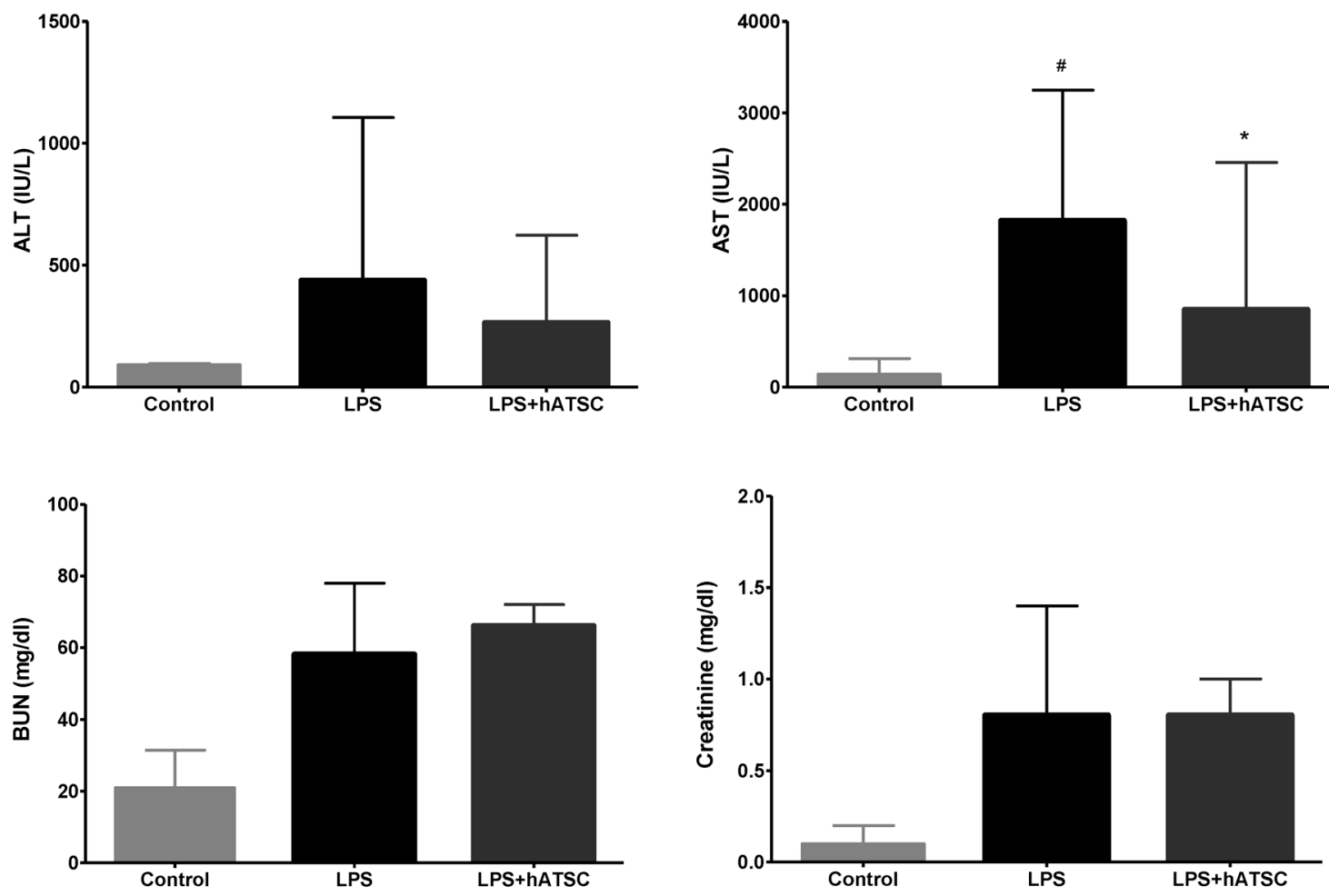

tions of the liver enzymes, ALT and especially AST, released into the circulation upon injury, were lower in the hATSC group than the saline group $(P<0.017)$. Whereas, kidney function, as measured by BUN and creatinine, did not differ between the groups. Lactate, an indicator of tissue hypoperfusion, tended to decrease in the hATSC group compared to the LPS group, but the difference between the groups was not statistically significant.

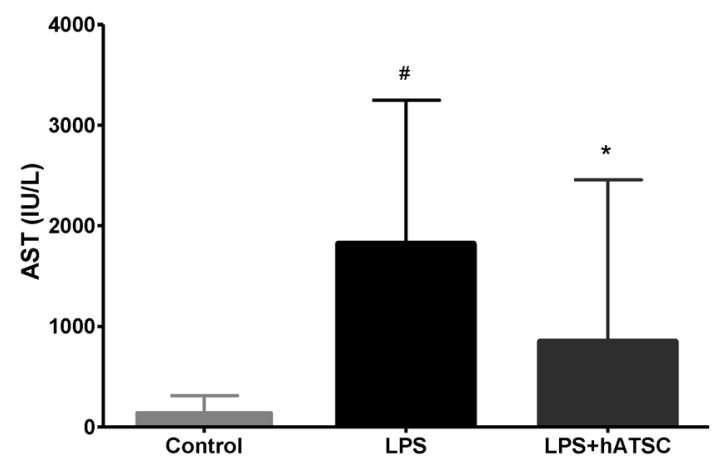

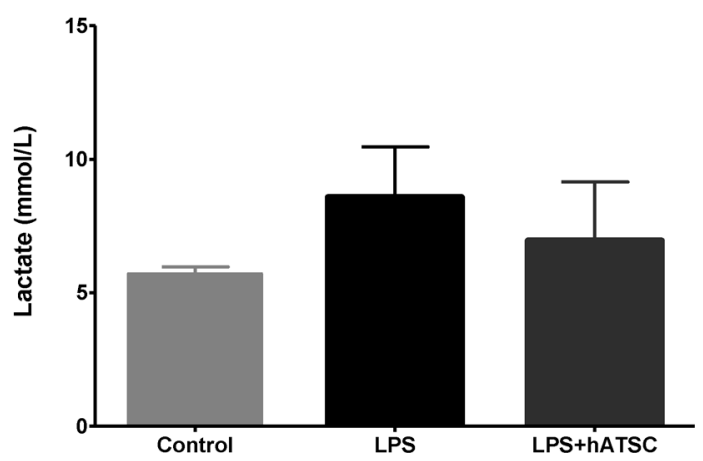

Figure 3. Effects of hATSC treatments on LPS-induced multi-organ dysfunction. Biomarkers of organ dysfunction (AST, ALT, BUN, creatinine, and lactate) were measured in serum. LPS increased the levels of each biomarker, demonstrating showed multi-organ injury. Administering hATSCs significantly reduced systemic AST levels (indicator of hepatic dysfunction) in endotoxemic rats $(P<0.017$ compared with LPS/saline group). ALT also tended to decrease with hATSC treatment. Kidney function (BUN and creatinine) did not differ between the saline- and hATSC-treated groups. Lactate (an indicator of tissue hypoperfusion) tended to improve in the hATSC treated group. $\# \mathrm{P}<0.017$, sham/saline vs LPS/saline group. ${ }^{*} \mathrm{P}<0.017$, LPS/saline vs LPS/hATSCs group. $\mathrm{n}=5$ per group. 


\section{Effect of hATSCs Treatment on LPS-induced Acute Lung Injury}

Lungs were sampled at $6 \mathrm{~h}$ after administering LPS. Lungs from animals receiving LPS and saline had congested alveolar capillaries, hemorrhage, inflammatory cell infiltration, and some alveolar wall thickening (Fig 4B). Lungs from animals treated with hATSCs were devoid of these changes (Fig 4C). To quantify the effects of hATSCs on lung injury, we used the Acute Lung Injury Score. The Acute Lung Injury Score was decreased in the hATSC group compared with the LPS group $(\mathrm{p}<0.017)$ (Fig 4D).

\section{hATSCs Treatment Protects the Apoptosis in Kidney}

In control rats, apoptotic cells (those with activated caspase-3) were rare in the kidney (Fig 5A), whereas, in endotoxemic rats, many more apoptotic cells were present (Fig 5B). hATSs treatment tended to reduce the number of apoptotic cells, compared with saline treatment (Fig 5C). The number of apoptotic cells stained red (TUNEL-positive) were counted in 4 randomly chosen fields per slide for all 3 groups. There were significantly fewer apoptotic cells in the hATSC treated group compared with the LPS-saline group $(\mathrm{p}<0.017)($ Fig $5 \mathrm{D})$

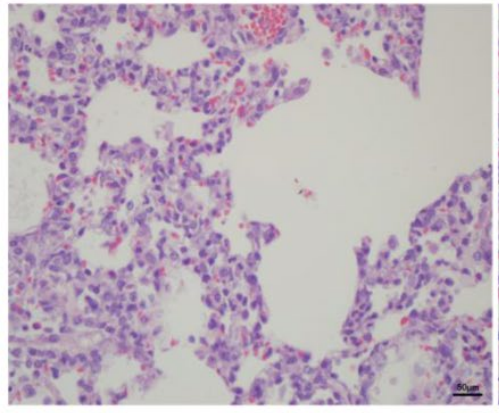

A. Control

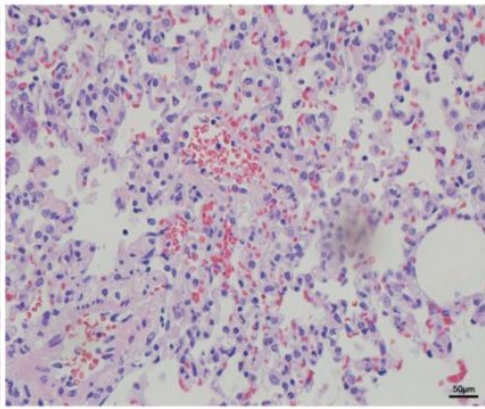

B. LPS

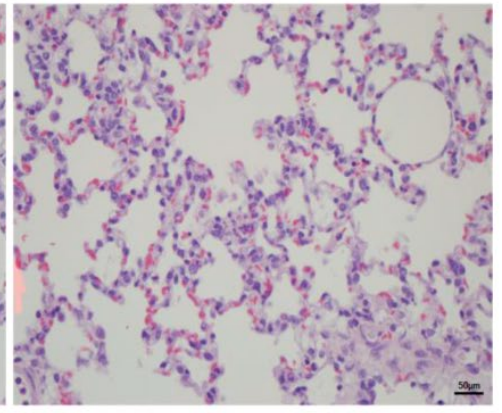

C. LPS+hATSCs

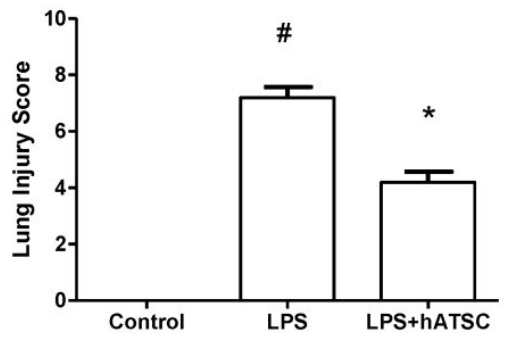

\section{Lung Injury Score}

Figure 4. Pulmonary histology by Hematoxylin-Eosin staining in Control (A), LPS (B), and hATSCs-treated LPS rats (C). $(\times 100)(B)$ Advanced inflammation with congestion of the alveolar capillaries, neutrophil infiltration, and diffuse thickening of the alveolar septum were observed in LPS group. (C) Neutrophil infiltration and thickening of alveolar septum were attenuated by hATSCs treatment. D. Acute Lung Injury Score was lower in the hATSCs-treated group $(4.2 \pm 0.8367)$ than the LPS-saline group (7.2 \pm 0.8367$)$. $\# P<0.017$, sham/saline vs LPS/saline group, $* \mathrm{P}<0.017$, LPS/saline vs LPS/hATSCs group. $\mathrm{n}=5$ per group. 


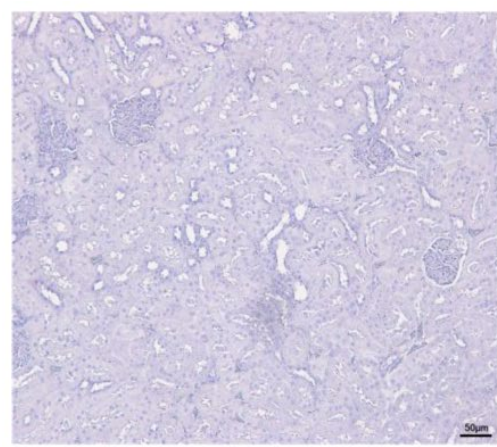

A. Control

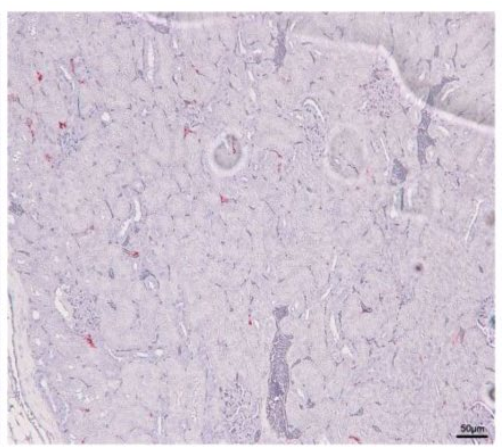

B. LPS

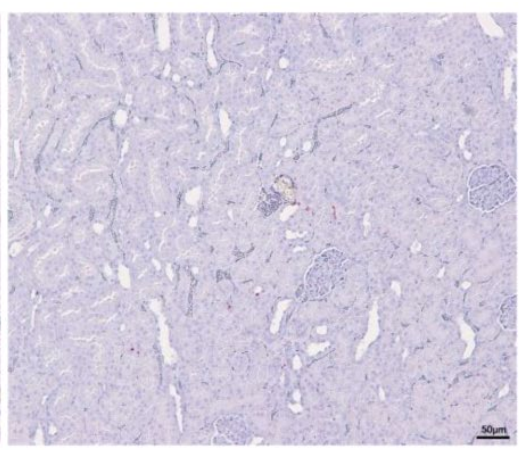

C. LPS+hATSCs

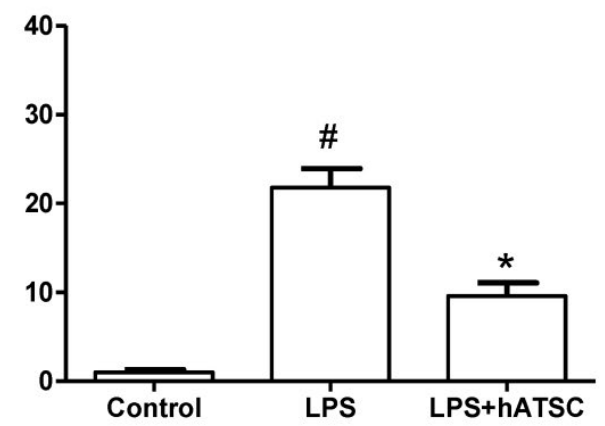

\section{The number of apoptotic cells}

Figure 5. hATSC treatment decreases apoptosis in the kidney after LPS challenge. TUNEL staining was used to identify apoptotic cells in kidney in the control (A), LPS (B) and LPS/hATSCs groups (C). $(\times 100)$. In control rats, apoptotic cells (TUNEL-positive nuclei stained red) were rare in the kidney, whereas, they were common in endotoxemic rats. hATSCs treatment reduced the number of apoptotic cells compared with the LPS/saline group. D. The total number of apoptotic cells (TUNEL-positive) cells were counted in 4 randomly chosen fields per slide cells in kidney. There were significantly fewer apoptotic cells in the hATSCs-treated group(9.6 \pm 3.209$)$ than the LPS-saline group $(21.8 \pm 4.764)$. $(\mathrm{P}<0.017)$. $\# P<0.017$, sham/saline vs LPS/saline group, ${ }^{*}<<0.017$, LPS/saline vs LPS/hATSCs group. $\mathrm{n}=5$ per group.

\section{Discussion}

The severity of sepsis is determined more by the specificity and ferocity of the host response than by the inciting organism. Uncontrolled inflammatory and coagulopathic mechanisms lead to endothelial and epithelial cell injury, apoptosis and cellular activation with increased production of anti-inflammatory mediators, leukocyte adhesion and transmigration, and activation of the coagulation and complement systems. Ironically, the inflammatory and coagulopathic mechanisms that are detrimental when uncontrolled during sepsis are probably beneficial on a normal day. Confined inflammation and accelerated coagulation are beneficial when they limit spread of local infection or injury[22].

Stem cells characteristic include self-renewal, long-term viability, and the potential to differentiate into diverse cell types depending on specific environmental conditions. This potential makes MSCs attractive candidates for cell therapy applications[23-25]. Attempts to use BMSCs therapeutically have been explored in preclinical and clinical studies of various immune associated diseases, such as ischemic heart disease, ischemic kidney injury, and Crohn's disease[26-32]. Also, several reports have demonstrated the therapeutic efficacy of BMSCs in animal models of sepsis and that BMSCs suppress systemic inflammation[8,33,34].

A critical issue for the clinical use of BMSCs, however, is the large quantities required for treatment. MSCs from subcutaneous adipose tissue were first identified by Zuk et al[11]. Since then, ATSCs have been studied as an alternative to BMSCs in sev- 
eral animal models of incurable diseases[26,36,37]. Unlike BMSCs, large amounts of ATSCs can be easily obtained from a disposable byproduct of lipoaspiration of healthy donors, and rapidly expanded in vitro to generate sufficient dosages[19,21,37]. Moreover, ATSCs have been shown to share immunomodulatory properties with BMSCs[19,36].

The beneficial effects of MSCs were mostly attributed to paracrine effects, the release of signaling factors for tissue repair, rather than incorporation into tissue, trans-differentiation, or fusion with cells in injured areas[38,39]. These paracrine effects have been attributed to immunosuppresive cytokines such as transforming growth factor- $\beta$ (TGF- $\beta$ ), hepatocyte growth factor, prostaglandin E2, IL-10, and NFkB $[34,40]$. Those soluble factors and products derived from MSCs, modulate immune responses, create an immunosuppressive microenvironment capable of modulating inflammation. Aggarwal et al. showed that MSCs decreased secretion of TNF-a by mature type 1dendritic cells[7]. Currently, the precise mechanisms by which MSCs act remain unclear, but MSCs seem to have effects at multiple levels, not just a single immune response pathway[26,35].

The fact that our results occurred in a short time frame also suggests that hATSCs have beneficial effects through paracrine activity, rather than cell differentiation or cell fusion.

Like other reports, we observed that hATSC treatment significantly decreased the levels of pro-inflammatory cytokines (TNF-a and IL-6) and attenuated inflammatory changes in the lungs in response to experimental endotoxemia[9,24,36,37].

Multi-organ failure contributes to mortality in sepsis. The lung is the most common organ involved in severe sepsis, rapidly resulting in respiratory insufficiency[22,41]. Perhaps, because the lung is the only organ to directly receive the entire cardiac output and to be thoroughly exposed to all inflammatory and coagulation abnormalities[41]. In this study, hATSC treatment significantly diminished the levels of pulmonary proinflammatory cytokines, especially IL-6, and reduced the acute lung injury score.

We observed that hATSCs improved multi-organ failure due to decreased pro-inflammatory cytokines and reduced apoptosis in the kidney. The liver enzymes(ALT,AST) and lactate as an indicator of tissue hypoperfusion were decreased in the hATSC group. This results suggests that, hATSCs seem to reduce the detrimental imbalanced inflammation through several pathways and at several levels, in contrast to what can be achieved by any single mediator .

Gonzalez-Rey et al. demonstrated that hATSCs decreased inflammatory cytokine levels and increased IL-10 levels in experimental colitis[37]. We observed similar reductions in pro-inflammatory cytokine levels, but systemic IL-10 was not increased by hATSC treatment. In contrast, systemic IL-10 decreased in the hATSC treatment group, though not significantly, and pulmonary IL-10 level did not differ among the 3 groups. Shirley also had reported similar results like us, decreased IL-10 in the BMSC-treated group and elevated IL-10 in saline-treated CLP septic mice[24]. This discrepancy of IL-10 results may be due to differences in study design. Ne'meth et al. studied the effects of BMSCs pretreatment, thus BMSCs were delivered 24 hours before CLP procedure[34]. IL-10 levels started to rise $3 \mathrm{hr}$ after BMSCs treatment, almost doubled by 6 hours, and were still elevated at $12 \mathrm{hr}$. Whereas, we measured IL-10 levels 6 hours after hATSC treatment, thus they may not have reached their maximum level. Therefore, further IL-10 increases might have been possible, given more time. In addition, Gonzalez-Rey et al used a higher ratio of hATSCs to animal weight $\left(1 \times 10^{6}\right.$ in mice, weight $\left.30 \mathrm{~g}\right)$ compared with our study $\left(2 \times 10^{6}\right.$ in rat, weight 250 300g)[37]. These differences in study design may result in conflicting observations of IL-10.

One limitation of this study is the endotoxemic animal model, which does not completely reflect septic conditions in humans. A variety of experimental animal models have been used to study sepsis pathophysiology and treatment[42]. We used an endotoxemia model instead of a polymicrobial sepsis model that may better mimic human sepsis. The reason we chose this model was because the primary experimental question was whether hATSC treatment would modulate inflammation and systemic inflammation abnormalities, especially early in sepsis, can be observed and quantified more quickly and reliably in animal models of endotoxemia[43]. Further studies to explore the effects of hATSCs in sepsis should consider polymicrobial models. Also, the timing of exogenous stem cell infusion after injury may be important, but the optimal time point has not yet been established. In this study, we infused hATSCs $30 \mathrm{~min}$ after inducing sepsis, which is not clinically possible. Therefore, future studies should explore the effects of hATSCs infused at different times after sepsis.

In conclusion, hATSC treatment in an endotoxemic rat model modulated host responses, decreased inflammatory cytokine levels in serum and lung, reduced alveolar inflammatory cell infiltration in the lung, and prevented apoptosis in the kidney in response to endotoxemia. hATSC administration also reduced liver injury and improved tissue hypoperfusion. A systemic hATSC injection at the disease onset 
ameliorated the serological and histological signs of endotoxemia and they could be attractive candidates for cell therapy to treat endotoxemia.

\section{Acknowledgements}

This study was supported by the Clinical Research Institute, Daejeon St. Mary's Hospital, Medical College of Catholic University and a grant from the Korea Healthcare Technology R\&D Project, Ministry for Health, Welfare\&Family Affairs, Republic of Korea. (SN: A092258 )

\section{Competing Interests}

The authors have declared that no competing interest exists.

\section{References}

1. Lolis E, Bucala R. Therapeutic approaches to innate immunity: severe sepsis and septic shock. Nat Rev Drug Discov. 2003;177(3):635-74.

2. Osuchowski MF, Welch K, Siddiqui J, Remick DG. Circulating cytokine/inhibitor profiles reshape the understanding of the sirs/cars continuum in sepsis and predict mortality. J Immunol. 2006;177(3):1967-74.

3. Monneret G, Venet F, Pachot A, Lepape A. Monitoring Immune Dysfunctions in the Septic Patient: A New Skin for the Old Ceremony. Molmed. 2008;14(1-2):64-78.

4. Tracey KJ, Fong Y, Hesse DG, Manogue KR, Lee AT, Kuo GC, et al. Anti-cachectin/TNF monoclonal antibodies prevent septic shock during lethal Bacteraemia. Nature .1987;330(6149):662-4.

5. Deans KJ, Haley M, Natanson C, Eichacker PQ, Minneci PC. Novel therapies for sepsis: a review. J Trauma. 2005;58(4):867-74.

6. Aggarwal S, Pittenger MF. Human mesenchymal stem cells modulate allogeneic immune cell responses. Blood. 2005;105(5):1815-22.

7. Aggarwal S, Fibbe WD. Immunomodulatory properties of mesenchymal stromal cells. Blood. 2007;110(10):3499-506.

8. Xu J, Woods CR, Mora AL, Joodi R, Brigham KL, Iyer S, et AL. Prevention of endotoxin-induced systemic response by bone marrow-derived mesenchymal stem cells in mice. Am J Physiol Lung Cell Mol Physio. 2007; 293(1):131-41.

9. Mei SH, Haitsma JJ, Dos Santos CC, Deng Y, Lai PF, Slutsky AS, et al. Mesenchymal stem cells reduce inflammation while enhancing bacterial clearance and improving survival in sepsis. Am j Respri Crit Care Med. 2010;182(8):1047-57.

10. Pittenger MF, Mackay AM, Beck SC, Jaiswal RK, Douglas R, Mosca JD, et al. Multilineage potential of adult human mesenchymal stem cells. Science. 1999;284(5411):143-7.

11. Zuk PA, Zhu M, Mizuno H, Huang J, Futrell JW, Katz AJ, et al. Multilineage cells from human adipose tissue: implications for cell-based therapies. Tissue Eng. 2001;7(2):211-28.

12. Chamberlain G, Fox J, Ashton B, Middleton J. Mesenchymal stem cells: their phenotype, differentiation capacity, immunological features and potential for homing. Stem Cells. 2007; 25(11):2739-49.

13. Horwitz EM, Prockop DJ, Fitzpatrick LA, Koo WW, Gordon PL, Neel M, et al. Transplantability and therapeutic effects of bone marrow-derived mesenchymal cells in children with osteogenesis imperfect. Nat Med. 1999;5(3):309-13.

14. Baksh D, Song L, Tuan RS. Adult mesenchymal stem cells: characterization, differentiation, and application in cell and gene therapy. J Cell Mol Med. 2004;8(3):301-16.

15. Barry FP, Murphy JM. Mesenchymal stem cells: clinical applications and biological characterization. Int J Biochem Cell Biol. 2004;36(4):568-84.

16. Dominici M, Hofmann TJ, Horwitz EM. Bone marrow mesenchymal cells: biological properties and clinical applications. J Biol Regul Homeost Agents. 2001;15(1):28-37.

17. Koc ON, Lazarus HM. Mesenchymal stem cells: heading into the clinic. Bone Marrow Transplant. 2001;27(3):235-9.

18. Dicker A, Le Blanc K, Aström G, van Harmelen V, gotherström C, BlomqviSt L, et al. Functional studies of mesenchymal stem cells derived from adult human adipose tissue. Exp Cell Res. 2005;308(2):28-90.
19. Yanez R, Lamana ML, Garcia-Castro J, Colmenero I, Ramirez M, Bueren JA. Adipose tissue derived mesenchymal stem cells have in vivo immunosuppressive properties applicable for the control of the graft-versus-host disease. Stem Cells. 2006;24(11):2582-91.

20. Imanaka $H$, Shimaoka $M$, Matsuura $N$, Nishimura $M$, Ohta N, Kiyono H. Ventilator-induced lung injury is associated with neutrophil infiltration, macrophage activation, and TGF-beta 1 mRNA upregulation in rat lungs. Anesth Analg. 2001;92(2):428-36.

21. Rigol M, Solanes N, Farre J, Roura S, Roque M, Berruezo A, et al. Effects of Adipose Tissue-Derived Stem Cell Therapy After Myocardial Infarction: Impact of the Route of Administration. J Card Failure. 2010;16(4):357-66.

22. Wheeler AP. Recent developments in the diagnosis and management of severe sepsis. Chest. 2007;132(6):1967-76.

23. Mansilla E, Marin G, Sturla F, Drago HE, Gil MA, Salas E, et al. Human mesenchymal stem cells are tolerized by mice and improve skin and spinal cord injuries. Transplant Proceed. 2005;37(1):292-4.

24. Shirley HJ, Mei SH, McCarter SD, Deng Y, Parker CH, Liles WC, et al. Prevention of LPS-Induced Acute Lung Injury in Mice by Mesenchymal Stem Cells Overexpressing angiopoietin1. PLos Med. 2007;4(9):e269.

25. McCulloch EA, Till JE. Perspectives on the properties of stem cells. Nat Med. 2005;11(10):1026-8.

26. Gupta N, Su X, Popov B, Lee JW, Serikov V, Matthay MA. Intrapulmonary delivery of bone marrow-derived mesenchymal stem cells improves survival and attenuates endotoxin-induced acute lung injury in mice. J Immunol. 2007;179(3):1855-63.

27. Le Blanc K, Rasmusson I, Sundberg B, Götherström C, Hassan M, Uzunel $\mathrm{M}$, et al. Treatment of severe acute graft-versus-host disease with third party haploidentical mesenchymal stem cells. Lancet. 2004;363(9419):1439-41.

28. Gnecchi M, He H, Melo LG, Noiseaux N, Morello F, de Boer RA, et al. Early beneficial effects of bone marrow-derived mesenchymal stem cells overexpressing Akt on cardiac metabolism after myocardial infarction. Stem Cells. 2009;27(4):971-9.

29. Lange C, Togel F, Ittrich H, Clayton F, Nolte-Ernsting C, Zander AR, et al. Administered mesenchymal stem cells enhance recovery from ischemia / reperfusion induced acute renal failure in rats. Kidney Int. 2005;68(4):1613-7.

30. Estrada EJ, Valacchi F, Nicora E, Brieva S, Esteve C, Froud T, et al. Combined treatment of intrapancreatic autologous bone marrow stem cells and hyperbaric oxygen in type 2 diabetes mellitus. Cell Transplant. 2008;17(12):1295-304.

31. Zappia E, Casazza S, Pedemonte E, Benvenuto F, Boanni I, Gerdoni E, et al. Mesenchymal stem cells ameliorate experimental autoimmune encephalomyelitis inducing T-cell anergy. Blood 2005;106(5):1755-61.

32. Garcia-Olmo D, Garcia-Arranz M, Herreros D, Pacsual I, Peiro C, Rodriguez-Montes JA, et al. A phase I clinical trial of the treatment of Crohn's fistula by adipose mesenchymal stem cell transplantation. Dis Colon Rectum. 2005;48(7):1416-23.

33. Gonzalez MA, Gonzalez-Rey E, Rico L, Busher D, Delgado M. Adipose-derived mesenchymal Stem cells alleviate experimental colitis by inhibiting inflammatory and autoimmune responses. Gastroenterology. 2009;136(3):978-89.

34. Nemeth K, Leelahavanichkul A, Yuen PS, Mayer B, Parmelle A, Doi K, et al. Bone marrow stromal cells attenuate sepsis via prostaglandin $\mathrm{E}(2)$-dependent reprogramming of host macrophages to increase their interleukin-10 production. Nat Med. 2009;15(1):42-9.

35. Krampera M, Sartoris S, Liotta F, Pasini A, Angeli R, Cosmi L, et al. Immune regulation by mesenchymal stem cells derived from adult spleen and thymus. Stem Cells Dev. 2007;16(5):797-810.

36. Puissant B, Barreau C, Bourin P, Clavel C, Corre J, Bousquet C, et al. Immunomodulatory effect of human adipose tissue-derived adult stem cells: comparison with bone marrow mesenchymal stem cells. $\mathrm{Br} \mathrm{J} H \mathrm{He}-$ matol. 2005;129(1):118-29.

37. Gonzalez-Rey E, Anderson P, González MA, Rico L, Büscher D, Delgado M. Human adult stem cells derived from adipose tissue protect against experimental colitis and sepsis. Gut. 2009;58(7):929-39.

38. M Gnecchi, Z Zhang, A Ni, Dzau VJ. Paracrine mechanisms in adult stem cell signaling and therapy. Circ Res. 2009;103(11):1204-19.

39. Lee JW, Fang X, Gupta N, Serikov V, Matthay MA. Allogeneic human mesenchymal stem cells for treatment of E. coli endotoxin-induced acute lung injury in the ex vivo perfused human lung. Proc Natl Acad Sci USA. 2009;106(38):16357-62.

40. Ryan JM, Barry F, Murphy JM, Mahon BP. Interferon-gamma does not break, but promotes the immunosuppressive capacity of adult human mesenchymal stem cells. Clin Exp Immunol. 2007;149(2):353-63. 
41. Rocco PR, Pelosi P. Pulmonary and extrapulmonary acute respiratory distress syndrome: myth or reality? Curr Opin Crit Care. 2008;14(1):50-5.

42. Doi K, Leelahavanichkul A, Yuen PS, Star RA. Animal models of sepsis and sepsis-induced kidney injury. J Clin Invest. 2009;119(10):2868-78.

43. Weil BR, Manukyan MC, Herrmann JL, Wang Y, Abarbanell AM, Poynter JA, et al. Mesenchymal stem cells attenuate myocardial functional depression and reduce systemic and myocardial inflammation during endotoxemia. Surgery. 2010;148(2):444-52. 